\title{
Managing Flexible Care With a Context Aware System for Ageing-In-Place
}

\author{
Saskia Robben ${ }^{1}$, Lilian Bosch ${ }^{1}$, Pascal Wiggers ${ }^{1}$, Jasmien Decancq ${ }^{2} \&$ Marije Kanis $^{1}$ \\ ${ }^{1}$ Amsterdam University of Applied Sciences \\ Amsterdam, the Netherlands \\ ${ }^{2}$ VUB - SMIT - iMinds \\ Brussels, Belgium
}

Contact: s.m.b.robben@hva.nl

\begin{abstract}
This paper describes the Care4Balance (C4B) system for better facilitating communication and task coordination between formal and informal caregivers, and older adults as care receivers. Field-tests with older adults $(n=3)$ and user studies ( $n=9)$ were conducted to evaluate the system and the perceived usefulness of the system. A review of related work and the study findings show that (1) the perceived benefit for the older target group was very low. The main motivation for using the system was triggered by the perceived benefit for their closest informal caregivers; (2) Informal caregivers do not regularly seek help for themselves, and (3) Introducing a C4B-like system is more than solving hardware and usability issues. The study suggests that more flexibility in the organizational structure of formal care (in The Netherlands and beyond) is needed.
\end{abstract}

Keywords-(in) formal care; aging-in-place; older adults; assistive technologies; Ambient Assisted Living (AAL)

\section{INTRODUCTION}

Due to the fast ageing population, more pressure is exerted on the demand for healthcare providers for older adults. The tendency for this group is to age independently at home (or 'age-in-place'), instead of going into assisted-living or nursing homes [1]. The care for these older adults who are living at home is often provided by (formal) professional healthcare providers and informal caregivers (family members or friends). The emotional involvement of informal caregivers in the wellbeing and safety of the older adult affects the motivation for doing the maximum for the care receiver, but can result in overprotecting the older adult, leading to an accelerated increase of dependency. Also, the demanding nature of caring can make it difficult for the informal caregiver to balance their care duties and personal lives, leaving them stressed and at higher risk of developing psychological illness [2]. The shift in the role of family member or friend to caregiver often happens gradually [3], and therefore they do not always consciously perceive themselves as informal caregivers. This makes it unlikely for them to be reached by informal care organisations that offer supportive resources, such as respite care [3]. While the role of these informal caregivers is becoming more and more important as there is an increasing shift from institutional care to home care [4], their interests are often ignored in discussions about the future of healthcare [1]. To address this lack, this paper discusses the literature and study of a system that aims to unburden the informal caregivers and empower the older adults.

\section{RELATED WORK}

In the field of Human Computer Interaction (HCI) and Pervasive Health, mitigating the burden of caring in the context of ageing-in-place is commonly approached from the perspectives of different stakeholders: the care recipients, the informal caregivers and (formal) professional healthcare providers.

Older adults generally value their independence and want to remain independent for as long as is manageable [3]. Explored approaches for helping to prolong this independence include increasing their self-sufficiency, by for example facilitating the self-management of their health (e.g. [5]) or improving their ways of interacting and communicating with others (e.g. [5], [6]). Ambient Intelligence (AmI) and Ambient Assisted living (AAL) focus on smart (sensor) technologies for helping older adults to continue living at home independently [7]. These systems positively contribute to unburdening caregivers by bringing peace of mind when regarding the safety of their care recipient. However, these often exclude the older adult as a user. With the exception of some examples (e.g. [8], [9]), most systems are generally focussed on presenting patterns of sensor data to informal caregivers or to professional healthcare providers [7]. Yet, care recipients have indicated a desire to have a say in what data about them is available, and to whom [9], [10].

Informal caregivers: Several studies (e.g. [11]) have shown the potential of using ICT systems for relieving informal caregivers that feel overburdened with their care responsibilities. These systems differ widely, showing that unburdening informal caregivers can be approached from different angles, such as providing social support (e.g. [12]) ambient monitoring (e.g. [4], [13]) and tools that assist with the management of care tasks (e.g. [14]). Although these examples show positive strategies for unburdening informal carers, little is known about how they are experienced by different stakeholders in practice.

For formal healthcare providers, the possibility of giving remote care to patients living at home has been explored. The goal of implementing remote care solutions are to provide more readily assistance, gain more knowledge about how a patient is doing and to seeing more patients (e.g. [15]).

In summative, most existing systems mainly focus on one or two of these stakeholders, but do not offer a complete solution that includes everyone involved in the care process. Ambient intelligence systems can provide benefits to multiple 
stakeholders, when deployed in a way that takes into account the older adult and its context. Therefore, this work focuses on a multi-layered system (including sensors) in-situ that aims to engage all stakeholders and deliver benefits for every stakeholder's interests. The most important concluded goals are: (1) to empower older adults: engage them in the use of the system, especially in controlling the information flow; (2) contribute to unburdening the informal caregivers.

\section{SYSTEM}

The system is developed in the context of the AAL project Care4Balance (C4B), in which researchers actively collaborated with care organizations and companies to ensure end user involvement and increase acceptance and adoption rates. The $\mathrm{C} 4 \mathrm{~B}$ system was designed to enable asynchronous communication and task (re-)distribution, to support relieving the burden of informal caregivers.

The interface for the older adults was aimed to be easy to use, taking into consideration that many older adults are not typically advanced computer users [16]. The front-end for older adults is a dedicated terminal that can be mounted on a fixed position (e.g. table) in the home of the older adults. The device has a touch screen and a build-in Radio-frequency identification (RFID) reader. In combination with a personalized set of RFID cards that represent various care tasks, the older person can interact with the system. This approach with physical cards was chosen to enable the older adults to interact in a more tangible way.

The home screen of the (web) application for (in)formal caregivers displays an overview of open- and assigned tasks, available people in the contact list, contextual information and a chat functionality for communication between caregivers. The caregivers can assign tasks to everyone, including themselves, which, after acceptance, can be placed in a calendar. This information is also presented on the front-end for older adults. Motion sensors in the home on key locations (e.g. bedroom) provide contextual information to the care network. With this information ('Is the person at home?', 'Is the person asleep?') the caretakers can be reassured while limiting privacy violation of the older participant.

\section{STUDY}

To study and evaluate how people perceive such a multistakeholder system in-situ, a field study $(n=3)$ was set up in Belgium and the Netherlands. In addition, shorter-term user tests $(n=9)$ were conducted.

\section{A. Methodology: field study}

The study's target participants were older adults with a care need living independently at home or in an assisted living facility. Internet connection in the homes of the older adults was provided. Their informal caregivers (at least two) were required to be proficient computer users with Internet access. The inclusion of formal caregivers was not required.

The participants were visited and interviewed at the start of the study and after each month. Participants were interviewed about their general attitudes towards the system and about its stability, usability and perceived usefulness. The actions that the participants performed with the system were also logged. The participants received a diary two weeks before the system was installed, so that possible changes in behavior could be recorded and so that they were more aware of all the care tasks to define the RFID cards afterwards.

Participants were recruited via care organizations and revalidation centers in the Netherlands and Belgium. The system was presented to professional caregivers who then suggested names of participants who met the inclusion criteria. To ensure that all studies were conducted in a uniform way, research manuals with walkthroughs and questionnaires for all appointments with the participants were prepared in advance and manuals for all components of the system were composed.

\section{B. Recruitment results}

The recruitment led to appointments with 42 care networks (older adults with care providers). The C4B system was explained to the older adult and / or their informal caregivers either in person or by phone by one or two researchers.

Only three care networks agreed to participate in the study. However, the motivations people gave for not participating provided valuable insights in people's perception with regards to their own situation and the C4B system.

Care network: Often, either the care receiver or the caregivers were willing to take part in the study, while the other party was not. Some older adults did not have informal caregivers.

Older adults: Many older adults felt that everything was already taken care of. They did not want to burden their informal caregivers by participating in a study. In addition, they were not used to ask for help from (in)formal caregivers if needed, as facilitated by the system. Instead, they were expecting help from formal caregivers at specific times.

The reactions to the possibility of sensor technology were mixed. Some found it reassuring and were open to its deployment, while others were concerned about their privacy and did not see the utility of this new technology. These findings are in line with other studies (e.g. [9]).

Some older adults felt that a telephone call was much easier than the indirect communication facilitated by the system. Also the cards gave some issues and finally, the front-end for older adults was perceived as a device that reminded them that they were in need of care.

Care providers: Some informal caregivers did not want to participate in the study, because they either lived too far away from the older adult, felt they were too busy, or thought that the older adult would not be able to use the new technology. Also, several informal caregivers did not perceive themselves as informal caregivers at all, saying that they 'just did some tasks for their relative'. The formal caregivers indicated that they were not used to care-on-demand. This indicates a larger issue that needs more attention and resulted in excluding the professional care providers from the first pilot.

\section{Field study findings}

Three care networks used the system for three months. One network decided not to have the sensors installed, while the other two networks recognized the value of the sensors. The 
context awareness provided peace of mind for the care network, but in response to some disruptions of the sensor system they indicated that the sensors should work consistently to not have the opposite effect. One participant found the dashboard unpleasant to use and switched to an iPad with the web application for caregivers. It took the third participant some time to get used to the terminal: after two months, she still had some difficulties controlling the system. However, presenting the cards did not seem to be a problem. The care receiver used the terminal for looking at her agenda of the day and for requesting help with certain tasks. The older participants did not feel that they could express themselves through the cards: "These actions are not what I need". The findings suggest that the cards should be more about irregular tasks rather than about recurring tasks. One of the older adults only used cards to indicate medicine intake.

The informal caregivers in one of the networks saw that all informal caregivers could see how much they are doing as an advantage. Others also stated that they would have found the system more useful if they would receive immediate notifications. During the pilot, the network did not change the way they organized care. Also, no new informal caregivers were added to the network. In all cases, the number of times people logged in decreased during the test period.

\section{User study}

The field study and the interviews in the recruitment phase provided insight in people's attitude towards the system. To gain more detailed feedback on how to improve the system, shorter-term user tests were conducted. The user study focused on the usability of the device for older adults and the perceived usefulness of the complete system. Inclusion criteria for the participants were: (1) the participant is at least 65 years of age; (2) the participant lives in his own home; (3) the participant has at least one informal caregiver; (4) the participant has good orientation of time and space. Three researchers, in teams of two, conducted the tests. A manual was prepared beforehand to ensure that all tests were conducted in the same way.

The procedure started with the participants being interviewed about their background and attitude towards technology. Next, the C4B system was explained, after which people were asked to execute five predefined scenarios with the system (e.g. 'make an appointment', 'find out who is available'). The actions of the participants were recorded on video, which was analyzed to identify usability issues. Finally, the participants were interviewed about their attitude towards the system.

Nine people ( 8 female, 1 male) participated in the user tests. The age of the participants ranged from 79 to 95 (mean 85). The participants all lived independently and almost all received formal and informal care. Five participants owned a computer, three also had Internet access.

\section{E. Findings of the user study}

Most participants $(n=7)$ indicated that, in general, they did not feel a need for new technology. Despite this attitude, the participants found the system usable. After some explanation and a short period of familiarization, they were all able to finish the tasks successfully. On a more detailed level, a number of usability issues came up. Some participants $(n=4)$ found the cards unclear and were afraid to lose the cards. It took the older adults more time than anticipated to complete a task, causing issues with the default system behavior of returning to the home menu after a timeout. Furthermore, the device adheres to interface conventions, such as an X-symbol for closing a screen and terms like 'ok' and 'cancel'. It also uses a hierarchical menu structure with which appointments, subtasks and the availability of caregivers can be found. Not all participants were familiar with these conventions.

After using the system, about half of the participants stated that the system did not have an added value for them. The other participants thought that there was an added value, but mostly towards their informal caregivers. The older adults themselves all preferred to use a telephone to contact their caregivers. Only one of the older adults stated that she would like to use the C4B system in daily life.

\section{F. General findings}

The C4B system was explicitly designed to empower care recipients by putting them in control of deciding which care tasks they needed. However, the older adults that participated in the studies did not feel this need. Some preferred arranging things simply by calling, while others did not want to burden their caregivers by asking them for help at all. When the researchers pointed out that the asynchronous communication might benefit their caregivers, who may not always be in a position to pick up the phone, and who can better divide tasks when they are visible for the whole care-network, the older adults stated that they would use the system if it helped to unburden their caregivers.

Part of the issue seems to be that many of the approached older adults felt that the device, designed as an easy to use alternative to mobile technology, uncomfortably amplifies that they are people in need of help. Ways to address this is that the device should either have other uses not related to care (e.g. tablet for making video calls with grandchildren) or be a more common-used item that does not have such associations (e.g. phone). The study further suggests that the solution people prefer, seems to depend on their computer literacy: older adults who have previous experience with computers were happy to accept a tablet as an alternative for the device, while computer-illiterate users would rather use a phone.

\section{DISCUSSION AND FUTURE WORK}

This work presented the design and evaluation of a multilayered system that aimed to engage and include each stakeholder's interests. The study of the system revealed several issues. Mainly, almost none of the older adults in the study saw an added benefit of the system for themselves.

One reason for this issue is stigmatization; the system putting too much emphasis on the (older) user as one in need of care. Part of the stigmatization might be addressed by putting more emphasis on reciprocity; someone who receives help with certain tasks, (e.g. getting out of bed and doing groceries), might be a caregiver in other contexts (e.g. by 
reading the newspaper to a neighbor). However, this requires a more active role of the care receiver in comparison with the current situation. Future system designs should take this into consideration, and should probably not make a distinction between care receivers and caregivers.

Future work could also further explore whether the device should be a familiar (tangible) item or should provide additional functionality to increase the system's acceptance. Adding extra functionality and mechanisms of reciprocity, on top of improving usability, might help to address issues from the perspective of the older adult. However, it seems that there are also broader issues at play.

The study findings suggest that the system does not fit with the way that people currently are organizing care. Achieving the goals that motivated the design of the system requires a different view on the way care is organized. The current protocols for care (in The Netherlands and Belgium) seem to leave little room for flexible task distribution between professional and informal caregivers. Healthcare is organized centrally, where clients receive a fixed amount of care based on their conditions. In addition, the informal caregivers are often not used to delegate tasks to others. As care policies are different internationally, one suggestion for future work is to repeat this study in different countries on a larger scale. Finally, 'care recipients' might be the wrong terminology, because older adults often indicate that they have difficulties with explicitly acknowledging and stating their care needs.

Furthermore, many of the care networks that were seen in this field study were relatively small. Consequently, there were only few people available that could possibly relief the main caregivers, and in some cases there were no informal caregivers at all. In these cases, the focus should rather be on ways to increase the care network. This may partially be achieved with digital tools. The field trial results demonstrate that introducing a C4B-like system is more than solving hardware and usability issues, it highlighted the way people think about what care is, or should be.

\section{ACKNOWLEDGMENT}

This research was part of the AAL-JP project Care for Balance, funded by the European Commision and ZonMW, and the COMMIT/ and Urban Vitality research programs, which are funded through the Dutch Ministry of Economic Affairs and HvA's Centre of Expertise. We thank our fellow project members and Chantal de Wit, Maartje van Diesen and Bas de Vleeschhouwer for their valuable contribution and our volunteers for their participation.

\section{REFERENCES}

E. Palm, "Who cares? Moral obligations in formal and informal care provision in the light of ICT-based home care.," Health Care Anal., vol. 21, no. 2, pp. 171-88, Jun. 2013.

S. Savage and S. Bailey, "The impact of caring on caregivers' mental health: a review of the literature.," Aust. Health Rev., vol. 27, pp. 111-117, 2004.
J. Van Exel, M. Morée, M. Koopmanschap, T. Schreuder Goedheijt, and W. Brouwer, "Respite care - An explorative study of demand and use in Dutch informal caregivers," Health Policy., vol. 78, no. 2-3, pp. 194-208, 2006.

F. Sadri, "Ambient intelligence: A survey," ACM Computing Surveys, vol. 43, no. 4. pp. 1-66, 2011.

[5] K. Daniel, C. L. Cason, and S. Ferrell, "Assistive technologies for use in the home to prolong independence," in Proc. 2nd Int. Conf. on PErvasive Technologies Related to Assistive Environments, 2009 , p. 26

J. Birnholtz and M. Jones-Rounds, "Independence and interaction: understanding seniors' privacy and awareness needs for aging in place," in Proc. SIGCHI Conf. on Human Factors in Computing Syst. (CHI '10), 2010, pp. 143-152.

[7] R. Landau, "Ambient intelligence for the elderly: Hope to age respectfully?," Aging health, vol. 9, pp. 593-600, 2013.

[8] K. C. Tseng, C. L. Hsu, and Y. H. Chuang, "Designing an intelligent health monitoring system and exploring user acceptance for the elderly," J. Med. Syst., vol. 37, no. 6, p. 9967, Dec. 2013.

M. Kanis, S. Robben, J. Hagen, A. Bimmerman, N. Wagelaar, and B. Kröse, "Sensor Monitoring in the Home : Giving Voice to Elderly People," in 7th Int. Conf. on Pervasive Computing Technologies for Healthcare (PervasiveHealth), 2013, pp. 97-100.

[10] M. Kanis, S. Alizadeh, J. Groen, M. Khalili, S. Robben, S. Bakkes, and B. Kröse, "Ambient monitoring from an elderly-centred design perspective: What, who and how," Proc. Int. Joint Conf. on Ambient Intelligence, pp. 330-334, 2011.

[11] H. Tellioğlu, M. Lewkowicz, A.F. Pinatti De Carvalho, I. Breškovic, S. Schinkinger, and M. Tixier, "Collaboration and coordination in the context of informal care (CCCiC): Concepts, Methods, and Technologies," in Proc ACM Conf. on Comput. Supported Cooperative Work and Social Computing (CSCW 2014), 2014, pp. 339-342.

[12] A. De Carvalho, S. Schinkinger, I. Breskovic, H. Tellioglu, A. Fabiano, P. De Carvalho, S. Schinkinger, I. Breskovic, and H. Tellio, "Technology for Work-Life Balance in Terms of Informal Care Work," in ECSCW 2013 Workshop "CSCW at the Boundary of Work and Life," 2013, pp. 1-6.

[13] L. Xiao, X. Yan, and A. Emery, "Design and evaluation of web interfaces for informal care providers in senior monitoring," in Proc. Annu. Meeting of Assoc. for Inform. Sci. and Technol. (ASIS\&T), 2013, vol. 50, no. 1, pp. 1-10.

[14] BrightStar Care, "Care Together," 2015. [Online]. Available: https://www.caretogether.com/.

[15] M. L. Lee and A. K. Dey, "Sensor-based Observations of Daily Living for Aging in Place," Pers. Ubiquitous Comput., vol. 19, no. 1, pp. 27-43, 2015.

[16] A. Dickinson, R. Eisma, and P. Gregor, "The barriers that older novices encounter to computer use," Univers. Access Inf. Soc., vol. 10, pp. 261-266, 2011. 